\title{
Blue surface-emitting distributed feedback lasers based on TPD-doped films
}

\author{
Eva M. Calzado, ${ }^{1,2}$ Jose M. Villalvilla, ${ }^{1,3}$ Pedro G. Boj, ${ }^{1,4}$ Jose A. Quintana, ${ }^{1,4}$ \\ Pablo A. Postigo, ${ }^{5}$ and María A. Díaz-García ${ }^{1,3, *}$ \\ ${ }^{1}$ Instituto Universitario de Materiales de Alicante (IUMA), Universidad de Alicante, 03080 Alicante, Spain \\ ${ }^{2}$ Departamento de Física, Ingeniería de Sistemas y Teoría de la Señal, \\ Universidad de Alicante, 03080 Alicante, Spain \\ ${ }^{3}$ Departamento de Física Aplicada, Universidad de Alicante, 03080 Alicante, Spain \\ ${ }^{4}$ Departamento de Óptica, Universidad de Alicante, 03080 Alicante, Spain \\ ${ }^{5}$ Centro Nacional de Microelectrónica, Consejo Superior de Investigaciones Científicas, \\ Isaac Newton 8, PTM Tres Cantos, 28760 Madrid, Spain \\ *Corresponding author: maria.diaz@ua.es \\ Received 28 September 2009; accepted 24 November 2009; \\ posted 15 December 2009 (Doc. ID 117696); published 15 January 2010
}

\begin{abstract}
Single-mode second-order distributed feedback (DFB) lasers with low threshold, based on polystyrene films doped with $30 \mathrm{wt}$ \% of the hole-transporting organic molecule N, $\mathrm{N}^{\prime}$-bis (3-methylphenyl)-N, $\mathrm{N}^{\prime}$ diphenylbenzidine (TPD) are reported. The laser emission wavelength was tuned between 415 and $427 \mathrm{~nm}$ by film thickness variation. The effectiveness of the DFB grating in improving the laser performance is evidenced by the observation of linewidths and laser thresholds lower than those of the amplified spontaneous emission characteristics shown by films without gratings. The use of holographic lithography as the technique for grating recording has allowed us to prepare large samples in a fast, versatile, and simple manner. (C) 2010 Optical Society of America
\end{abstract}

OCIS codes: $\quad 140.0140,140.2050,140.3580,160.0160,160.3380,160.4890$.

\section{Introduction}

Since the discovery of stimulated emission in semiconducting polymer films [1-3], extensive research has been devoted to the development of solid-state lasers based on semiconducting materials, including polymers, small organic molecules, oligomers, and dendrimers [4-6]. A very unique property of organic materials is that, due to their broad photoluminescence (PL) spectrum, the laser wavelength can be tuned over a wide range [7]. Among the various types of organic material, those that are semiconducting open the possibility of electrical pumping. Moreover, those that are soluble have the additional advantages of easy processing in the form of thin films

0003-6935/10/030463-08\$15.00/0

(C) 2010 Optical Society of America by inexpensive techniques and high compatibility with the substrates over which they are deposited. These characteristics open ways to heterogeneous integration of optic and electronic devices. One of the main challenges in the use of new active organic materials for lasing is the achievement of laser thresholds, low enough to allow pumping with compact and inexpensive optical sources [8-10].

The easiest way to evaluate the potential of a certain material to be used as an active laser medium consists in characterizing its amplified spontaneous emission (ASE) when deposited as thin films in a waveguide configuration $[2,11]$. In addition, this technique is the most convenient for comparing the performance of different materials, since it allows separating the variations due to the material from those due to the resonant cavity. The laser properties of 
films of a wide variety of molecular semiconducting materials have been reported in the literature [6,12-22]. In most cases, laser activity is observed at thresholds much lower than those of traditional dyes [23] and comparable to those of typical semiconducting polymeric laser materials $[4,6]$. The main reason is that molecular semiconducting materials can be doped into films at large concentrations, typically between 10 and $30 \mathrm{wt}$. \%, or even higher, without getting PL quenching. Note that the typical concentrations used with dyes are around 1wt.\%. For some particular molecular systems, ASE was observed even in neat films (nondiluted in an inert matrix). Examples of such materials are various thiopheneS,S-dioxide oligomers [16], spiro-type molecules [17], and the hole-transporting N,N'-bis (3-methylphenyl)-N, $\mathrm{N}^{\prime}$-diphenylbenzidine system (TPD) [12,24-27] (Fig. 1). Since film quality and supramolecular organization play a major role in obtaining high PL efficiencies and stimulated emission in the solid state, recent investigations of the ASE performance as a function of the concentration of the active molecule in the film have been reported for different materials [13,14], including TPD [27]. This way, the material characteristics that lead to the best performance (lower ASE threshold, narrower ASE linewidth, and larger tunability of the ASE wavelength) could be determined prior to the introduction of a laser cavity. For TPD, ASE thresholds as low as $1 \mathrm{~kW} / \mathrm{cm}^{2}$ have been previously measured when doped at $30 \mathrm{wt} . \%$ into polystyrene (PS) [27]. This value is one of the lowest reported in the literature

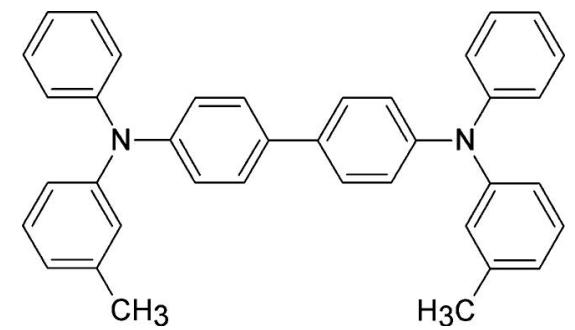

Fig. 1. Chemical structure of $\mathrm{N}, \mathrm{N}^{\prime}$-bis (3-methylphenyl)-N, $\mathrm{N}^{\prime}$ diphenylbenzidine (TPD).

for molecular materials, without energy-transfer processes being involved. Moreover, it is comparable to thresholds of typical semiconducting polymers emitting in the blue region of the optical spectrum (see comparison in Table 1) [17,27-31]. Note that thresholds in Table 1 are given in power density units $\left(\mathrm{kW} / \mathrm{cm}^{2}\right)$, which are the most convenient to compare the performance of different materials [15].

One of the simplest laser resonators consists in placing the active medium between two mirrors, which is the method typically used with dyes $[6,17]$. Other configurations include vertical microcavities $[3,4,6]$, whose thresholds are generally large, microrings, and microdisks $[4,6]$. Although the two latter devices generally show low thresholds, their interest for applications is limited by the fact that they do not provide a well-collimated beam of light. An alternative consists of using a distributed feedback (DFB) cavity in which a periodic modulation of the refractive index by a surface grating (on the substrate or on the active medium) is made in

Table 1. Comparison of the ASE Performance of Various Semiconducting Organic Materials Emitting in the Blue Region of the Optical Spectrum

\begin{tabular}{|c|c|c|c|c|c|}
\hline Material & Chemical Structure $^{a}$ & $\lambda_{\mathrm{ASE}}^{b}$ & $I_{\text {th-ASE }}{ }^{c}$ & Type of Sample $^{d}$ & Ref. ${ }^{e}$ \\
\hline $30 \mathrm{wt} . \%$ TPD-doped PS film & & 417 & 1 & $\mathrm{SC}$ & {$[\underline{27}]$} \\
\hline Spiro-6P neat film & & 425 & 3 & $\mathrm{SC}$ & [17] \\
\hline BP1T & $\begin{array}{l}\Rightarrow 0 \\
\Rightarrow 0\end{array}$ & 462 & 13 & $\mathrm{C}$ & {$[\underline{28}]$} \\
\hline CCN-DPDSB & & 469 & 39.5 & $\mathrm{C}$ & [29] \\
\hline $\mathrm{BPCz}$ neat film & & 421 & 4 & $\mathrm{SC}$ & [30] \\
\hline PFO neat film & & 466 & 2 & $\mathrm{SC}$ & [31] \\
\hline
\end{tabular}

${ }^{a}$ Chemical structure of the active molecule.

${ }^{b} \lambda_{\text {ASE }}$, ASE wavelength (nm).

${ }^{c} I_{\text {th-ASE }}$, pump intensity threshold for ASE observation $\left(\mathrm{kW} / \mathrm{cm}^{2}\right)$.

${ }^{d}$ Type of sample: SC, spin coating; C, single crystal.

${ }^{e}$ Ref., reference. 
order to obtain light reflected by "Bragg-scattering" $[32,33]$. In DFB lasers, light propagating in a waveguide mode is scattered by the periodic corrugation, so the scattered light from each corrugation combines coherently and an new Bragg-scattered wave is formed, propagating in a new direction. Lasing occurs close to the so-called Bragg wavelength $\left(\lambda_{\text {Bragg }}\right)$ given by

$$
m \lambda_{\text {Bragg }}=2 n_{\text {eff }} \Lambda,
$$

where $n_{\text {eff }}$ is the effective refractive index of the waveguide, $\Lambda$ is the period of the grating, and $m$ is the order of diffraction. For first-order diffraction $(m=1)$, light is diffracted out to the edge of the film, while for the case of second-order diffraction $(m=2)$, light is diffracted out near perpendicular to the plane of the waveguide [34]. DFB structures present several advantages, such as single-mode emission (important for a number of applications), easy deposition of the organic film, low thresholds, and no need of mirrors. Numerous examples of optically pumped DFB organic lasers have been published in the literature $[\underline{4}, \underline{6}]$. Various methods for grating recording, i.e., holography, electron-beam lithography, and soft lithography have been used.

In this work we have built second-order DFB lasers based on $30 \mathrm{wt}$. \% TPD-doped PS films as active laser material. Several reasons support our interest in TPD-based devices: first, the low ASE thresholds of TPD-based films; second, the fact that TPD emits in the blue part of the spectrum, where the number of available sources is limited; and third, our capability of controlling the emission characteristics of TPD by the knowledge provided by our previous ASE studies [12,25-27]. DFB gratings have been fabricated by holographic lithography (HL), using dichromated gelatin (DCG) as photoresist, due to its very good resolution capability [35,36]. This HL technique has several advantages: it is fast and versatile, no master is needed, and it allows preparation of large samples.

\section{Sample Preparation and Experimental Procedures}

DFB lasers were made by deposition of polymer active films on top of glass substrates over which surface relief gratings were previously recorded by HL $[35,36]$, followed by conventional reactive ion beam etching (RIBE). An argon laser operating at $364 \mathrm{~nm}$ was used to record a light intensity pattern over a $700 \mathrm{~nm}$ thick spin-coated film of the photosensitive material DCG. The pattern was obtained by the interference of the direct beam with the beam reflected in a mirror attached to the sample holder (see Fig. 2). An absorbent glass was attached to the back side of the plate with an index matching liquid to avoid backward reflections from the surface of the glass support. The geometric parameters for grating recording were carefully adjusted in order to get a $\Lambda$ value close to $270 \mathrm{~nm}$, which satisfies Eq. (1) for $m=2, n_{\text {eff }} \sim 1.554$ (for a 30 wt. \% TPD-doped PS film), and $\lambda=420 \mathrm{~nm}$. Relief gratings in the DCG

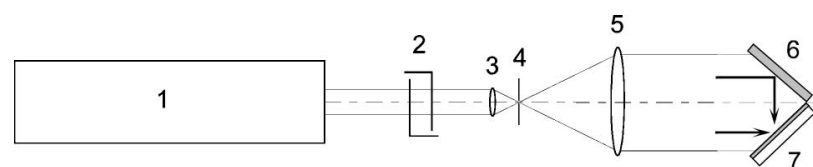

Fig. 2. Experimental setup for holographic recording of gratings over DCG: 1, argon laser; 2 , shutter; 3 , microscope objective; 4, pinhole; 5 , collimator; 6 , mirror; 7 , DCG film over glass.

film were then obtained by dry development in an oxygen electron cyclotron resonance stream. These gratings were then transferred to the glass substrate by RIBE with $\mathrm{CHF}_{3}$ gas, and the residual resist was washed off.

The active films were spin coated over the glass substrates with gratings from toluene solutions containing PS as an inert polymer and $30 \mathrm{wt}$. \% (with respect to PS) of TPD. Films with different thicknesses were prepared by adjusting the percentage of PS with respect to the solvent (between 2.5 and 6 wt. \%). Film thickness was measured with an interferometer coupled to an optical microscope. To properly assess the effect of the grating in the emission characteristics, we also deposited films of similar characteristics over substrates without gratings.

For the characterization of the emission properties of the DFB lasers, as well as of the reference films deposited over substrates without gratings, samples were illuminated with a pulsed $\mathrm{Nd}$ :YAG laser (10 ns, $10 \mathrm{~Hz}$ ) operating at $355 \mathrm{~nm}$, which lies close to the maximum absorbance of TPD [12,27]. The energy of the pulses was varied using neutral density filters. The pump laser beam was expanded and collimated, and only the central part was selected in order to ensure uniform intensity. The pump beam was incident upon the samples at $\sim 20^{\circ}$ with respect to the normal to the sample plane, and it formed a circular spot with a diameter of $1.3 \mathrm{~mm}$. The emitted light was collected normal to the surface with a fiber spectrometer placed at $1 \mathrm{~cm}$ of the sample.

\section{Results and Discussion}

Figure 3 shows a scanning electron microscopy (SEM) image of the grating recorded over the DCG film and an atomic force microscopy (AFM) image of the grating once transferred to glass. A period of $\Lambda=270 \mathrm{~nm}$ $( \pm 2 \mathrm{~nm})$ and a depth of $70 \mathrm{~nm}( \pm 15 \mathrm{~nm})$ were obtained. The diffraction efficiency $(\eta)$ of the gratings over DCG and over glass was $28 \%$ and $6 \%$, respectively. PS films containing $30 \mathrm{wt}$. \% of TPD, deposited by spin coating over the glass substrates with gratings, formed active waveguides with thicknesses varying between 150 and $300 \mathrm{~nm}$.

Figure 4 shows the emission spectra at low and high pump intensities for a DFB device with a $250 \mathrm{~nm}$ thick PS/TPD film [Fig. 4(a)], as well as for a sample consisting of a similar film but deposited over a glass substrate without a grating [Fig. 4(b)]. In this latter case, no real lasing takes place since no cavity is present. Nevertheless, gain narrowing at a certain pump intensity due to ASE is observed, as already discussed in detail in previous works 


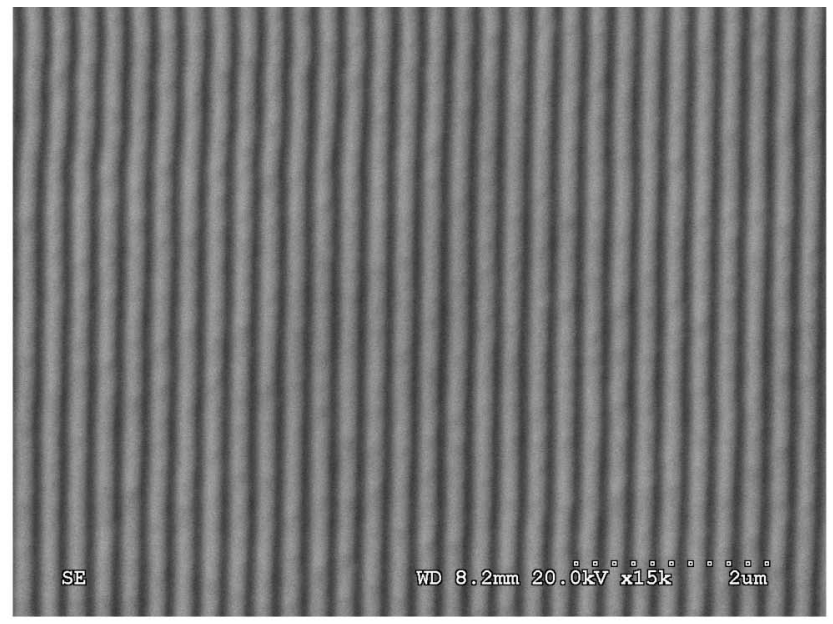

(a)
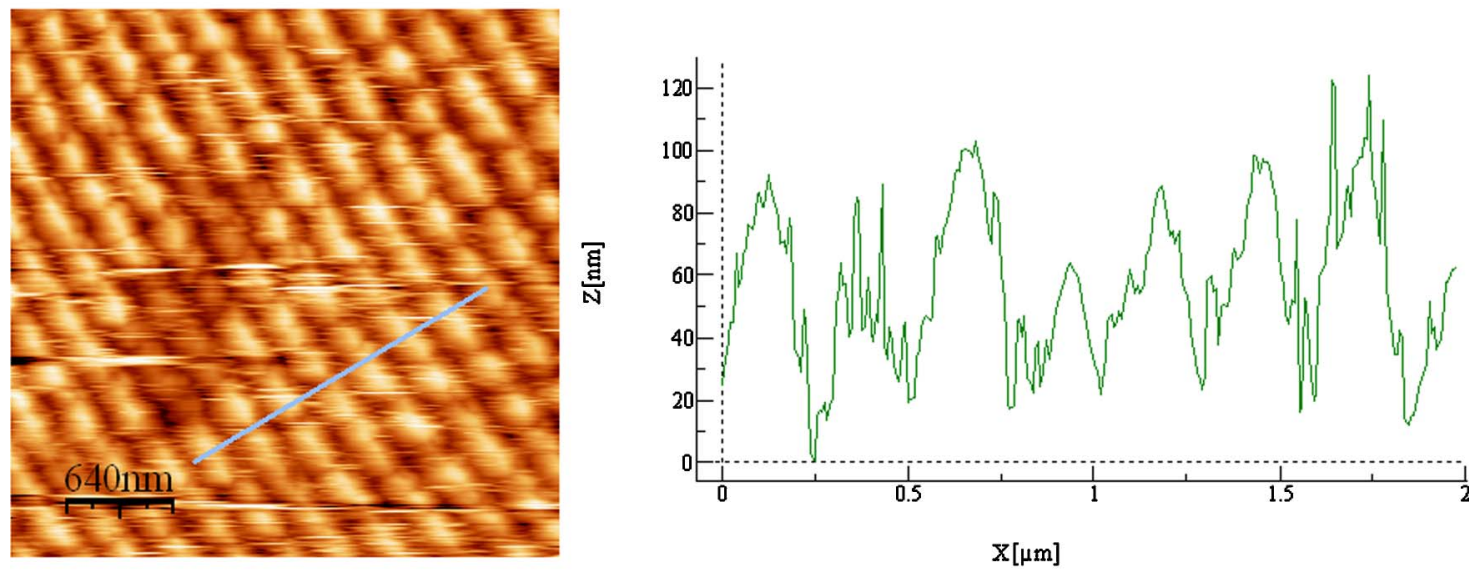

(b)

Fig. 3. Photographs of the relief gratings recorded in (a) a DCG film and (b) in glass after the transfer by RIBE, obtained by SEM and AFM, FMrespectively.

[25-27]. By comparing Figs. $\underline{4(\mathrm{a})}$ and $\underline{4(\mathrm{~b})}$, it is clear that the presence of the grating leads to changes in the emission spectra. At low pump intensity, the output spectrum of the DFB device is broad and rather different than the one obtained with the sample without a grating. In accordance with other authors [34], the modifications observed by the presence of the grating are attributed to waveguided PL that has been Bragg scattered out of the waveguide at an angle normal to the substrate. It is also observed that the spectra of the DFB device exhibit a characteristic dip in the emission intensity at around $\lambda=417 \mathrm{~nm}$, due to an inhibition of the propagation of the waveguided light by the grating. This Bragg dip can be envisaged as a photonic stop band for waveguided modes [34]. At high pump intensity, DFB lasing occurs at the long-wavelength edge of the Bragg dip [see Fig. 4(a)] and a narrow peak dominates the emission. This particular device (thickness $250 \mathrm{~nm}$ ) operates at $418 \mathrm{~nm}$ with a linewidth [defined as the full width at half-maximum (FWHM)] lower than $2 \mathrm{~nm}$, limited by the resolution of our spectro- meter. The emission is strongly polarized, with the electric field vector parallel to the DFB grooves.

In the case of the film deposited over a glass substrate without a grating [Fig. 4(b)], the spectrum obtained at low pump intensity is similar to that recorded in a fluorimeter. This latter one consists of a main band with a maximum at $401 \mathrm{~nm}$ and a first vibronic peak at $420 \mathrm{~nm}$ [27]. Although the emission spectrum obtained with the characterization setup used in this work also shows two peaks, their relative intensities are somewhat different than the ones obtained in the fluorimeter, and a slight red shift of the maximum of the main band is observed. These differences are simply due to the different geometry used to collect the emitted light in the fluorimeter and in the setup for the DFB characterization [27]. Despite these slight spectral changes, the most important aspect to consider here is the fact that a dip, similar to that observed in Fig. 4(a) for the sample with grating, is not observed. With respect to the high pump intensity spectrum, due to ASE, its linewidth is clearly reduced (up to around $5 \mathrm{~nm}$ ) with respect to the 

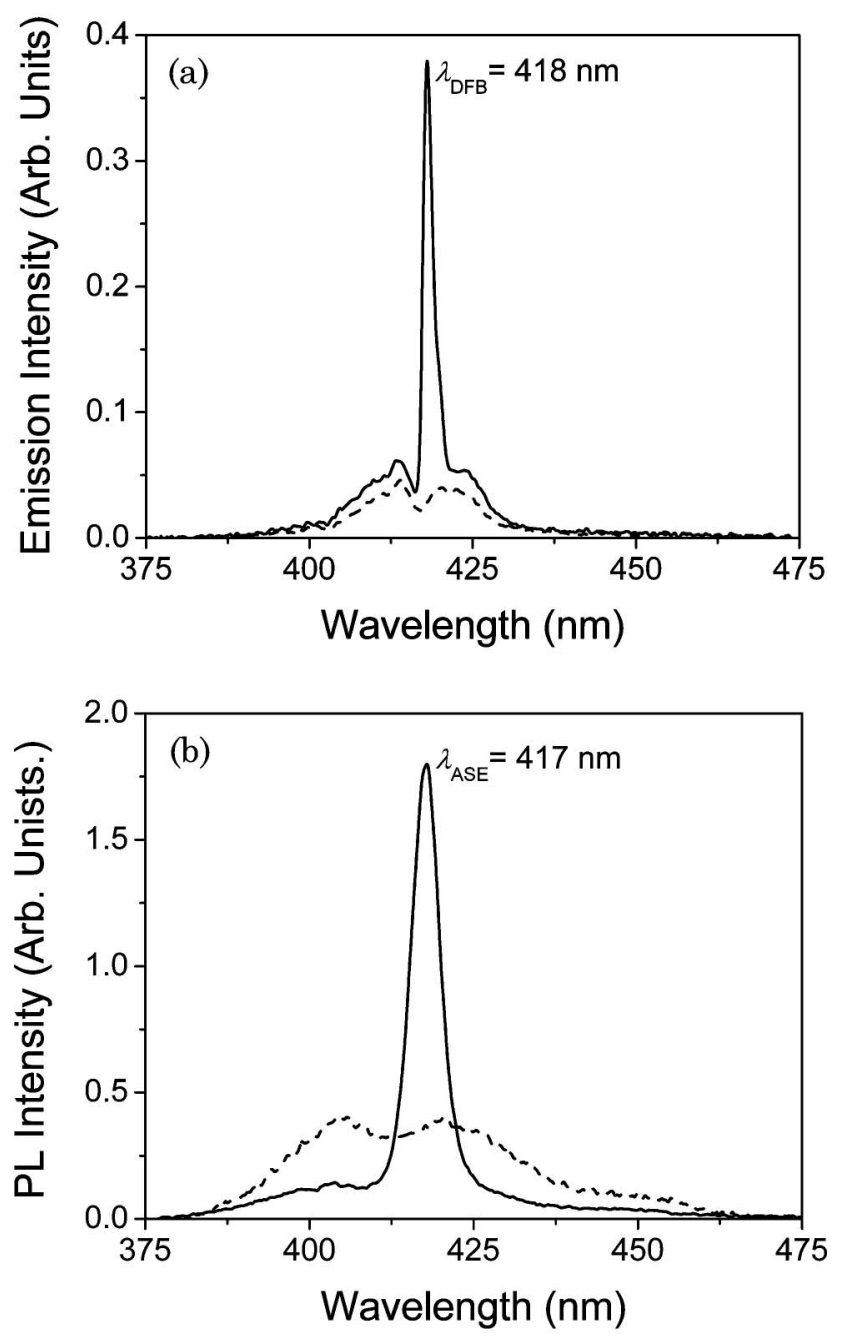

Fig. 4. Emission spectra of a TPD-doped PS film deposited on glass (a) with and (b) without DFB grating, at low $(1 \mu \mathrm{J} /$ pulse, dashed curve) and high ( $20 \mu \mathrm{J} /$ pulse, solid curve $)$ pump intensity.

low pump intensity one, and its total output intensity (at $417 \mathrm{~nm}$ ) is drastically increased. Note that the obtained ASE linewidth is larger than that measured with the DFB structure $(<2 \mathrm{~nm})$.

To understand the mechanism that controls laser emission, it is useful to analyze in more detail the DFB laser spectrum [Fig. 4(a)]. In general, DFB lasers made with a periodic modulation in the refractive index normally lase in two modes, one slightly below $\lambda_{\text {Bragg }}$ and the other slightly above it [32-34,37]. Single-mode DFB lasers can be made by introducing a phase shift in the periodic modulation or by modulating the gain instead of the refractive index. Gain modulation adds an imaginary part to the coupling coefficient; thus, laser emission is observed inside the photonic stop band [38,39]. As observed in Fig. 4 (a), for our DFB device, single-mode operation is evidenced by the observation of a lasing peak on the longwavelength band edge of the PL spectrum. In fact, for all the DFB devices fabricated in this work and emitting at different wavelengths (described latter in this section), DFB emission was observed on the long- wavelength side of the stop band. The inhibition of the lasing mode below $\lambda_{\text {Bragg }}$ can be explained, as suggested by other authors [34,40-43], by the fact that the cavity losses for this band edge are lower than for the short-wavelength one, so the long-wavelength mode has a lower threshold [34]. According to this idea, the mechanism dominating laser emission in our DFB lasers would be index modulation. Other authors have attributed the observation of singlemode operation in polymer lasers to the effect of periodic gain modulation, rather than index modulation $[38,39,41-43]$. In those systems, lasing was observed very close to $\lambda_{\text {Bragg }}$ (inside the stop band) and that was explained in terms of the periodically varying thickness of the polymer layers. While periodic gain modulation might be present in our DFB lasers, the fact that the laser mode was found to oscillate on the band edge, and not in the gap, strongly points to a system dominated by index modulation from the permanent grating structure.

Figure 5 shows the evolution of the DFB laser intensity at $\bar{\lambda}_{\text {lasing }}$ and of the ASE intensity at $\lambda_{\mathrm{ASE}}$ with the increasing pump intensity, for the samples described in Fig. 4. For the DFB laser, a nearly exponential increase is clearly observed for pump intensities above $6 \mu \mathrm{J} /$ pulse, the value identified as the laser threshold. For the sample without grating, a change in slope is not clearly defined; thus, the numerical value of the ASE threshold $(12 \mu \mathrm{J} /$ pulse $)$ was obtained by determining the intensity at which the FWHM of the emitted light decays to half of its maximum value. Here, thresholds are given in energy per pulse units, instead of in power density units, due to the difficulties of properly calculating thresholds in these latter units when using the DFB characterization setup. This issue is discussed in more detail below.

The ASE results obtained for the film without a grating are similar, in terms of linewidth and

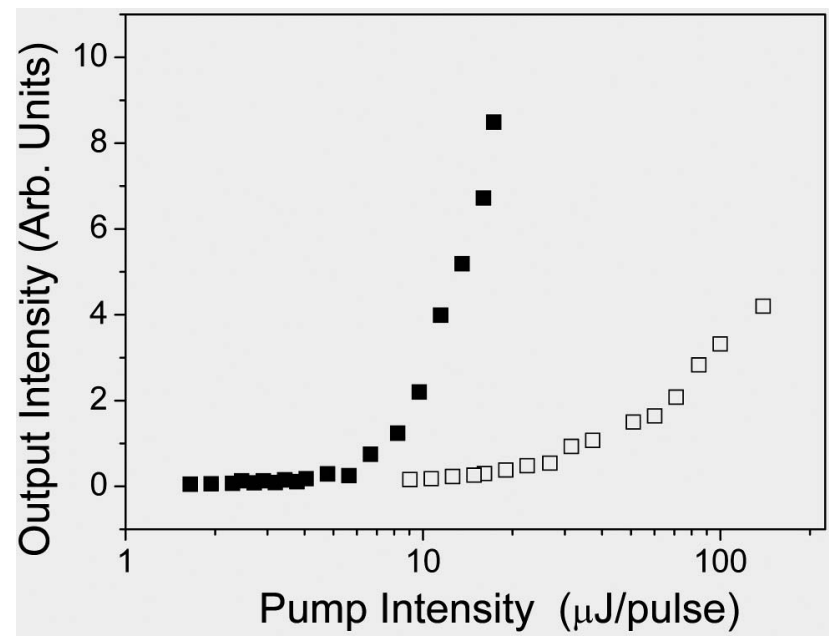

Fig. 5. Output intensity at $\lambda_{\mathrm{DFB}}=418 \mathrm{~nm}$ and at $\lambda_{\mathrm{ASE}}=417 \mathrm{~nm}$ as a function of pump intensity for a TPD-doped PS film deposited over a glass substrate with (solid squares) and without (open squares) a second-order DFB grating, respectively. 
emission wavelength, to those obtained in the setup typically used for the ASE characterization [27], where a stripe of light, instead of a spot, is used to excite the sample. However, it should be noted that the thresholds measured in each setup are rather different, mainly due to the different size of the pump beam. Experiments performed with waveguides characterized in the typical ASE setup for various pump stripe lengths (unpublished data), have shown that the ASE threshold decreases with the length of the pump stripe, saturating for stripe lengths above $2 \mathrm{~mm}$. Note that, in the typical ASE setup, the size of the pump stripe is $3.5 \mathrm{~mm}$. Dependencies of the ASE thresholds with the length of the pump stripe have also been observed by other authors, in that case with a poly(phenylene vinylene) derivative [44]. Because in our DFB setup a spot of only $1.3 \mathrm{~mm}$ in diameter is used, the thresholds determined are not the optimized ones. Increasing the size of the spot in order to approximate the conditions of the ASE setup would result in difficulties in recollecting the light with the optical fiber. Thus, we decided to express the thresholds measured in the DFB setup in energies per pulse and use a similar film without grating to assess the effect of the grating on decreasing the threshold. All the considerations just stated justify the lack of coherence in the threshold values found in the literature $[2, \underline{8}, \underline{31}, \underline{34}, \underline{38}, \underline{40}-47]$. Very often, the ASE thresholds reported for certain materials are apparently lower than the laser thresholds measured for the same materials inside a resonant cavity. These inconsistencies are often due to the different setups used to characterize the properties. Moreover, as already discussed in the introduction, for a proper analysis of the potential of different types of materials for laser purposes, ASE thresholds, rather than laser thresholds, should be compared. In this latter case, it would not be clear whether the differences in threshold are due to the material or to the cavity.

The tuning of the DFB emission wavelength over the TPD emission spectrum was investigated by preparing and characterizing devices with different film thicknesses. Results are shown in Table 2. In all cases, a single DFB peak was observed, given that all the films studied supported one waveguide mode.

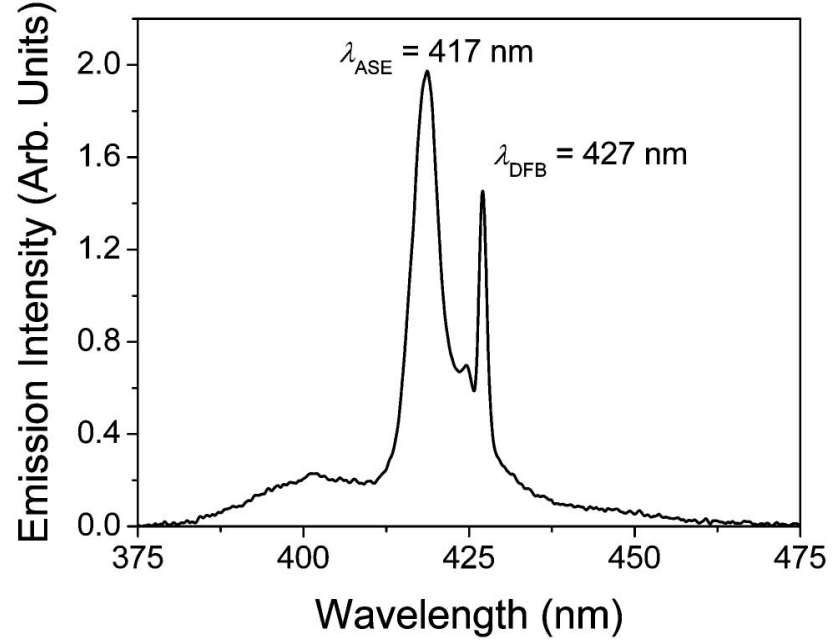

Fig. 6. Emission spectrum of a second-order DFB laser based on a TPD-doped PS film working at $\lambda_{\mathrm{DFB}}=427 \mathrm{~nm}$.

This is in contrast with other works published in the literature $[37,48]$, dealing with devices based on active films thick enough to support more than one waveguide mode, in which several DFB peaks were observed. Concerning the emission wavelength of the DFB devices prepared in this work, it has been tuned from 415 to $427 \mathrm{~nm}$. The lowest thresholds were obtained for the devices emitting at 418 and at $415 \mathrm{~nm}$, with values of 6 and $11 \mu \mathrm{J} /$ pulse, respectively. These wavelengths are close to the vibrational peak of the PL spectrum, at which ASE takes place. For devices emitting at wavelengths farther away from the ASE wavelength, i.e., $\lambda_{\mathrm{DFB}}>420 \mathrm{~nm}$, much larger pump intensities were needed in order to obtain laser emission. Moreover, a peak due to ASE is also observed. This is illustrated in Fig. 6 for the device whose laser emission appears at $427 \mathrm{~nm}$. Because of the competition of both processes, a precise determination of the laser thresholds and isolated laser DFB emission was not possible. These results indicate that, with these devices, the achievement of DFB laser emission in a wide range of wavelengths at reasonable thresholds is limited by the presence of ASE emission. Further experiments aiming to clarify the relevant parameters involved in the interplay between ASE and DFB are currently being performed.

Table 2. Laser and ASE Performance of $30 \mathrm{wt}$ \% TPD-Doped Polystyrene Films of Various Thicknesses Deposited over Glass with and without a Second-Order Distributed Feedback Grating

\begin{tabular}{lccccc}
\hline Substrate & $h^{a}$ & $\lambda_{\text {DFB }}{ }^{b}$ & $\lambda_{\text {ASE }}{ }^{c}$ & $I_{\text {th-DFB }}{ }^{d}$ & $I_{\text {th-ASE }}{ }^{e}$ \\
\hline Bare glass & 250 & - & 417 & & 11 \\
Glass with DFB grating & 160 & 415 & - & - & - \\
& 250 & 418 & - & - & - \\
& 275 & 423 & 417 & - \\
\hline
\end{tabular}

\footnotetext{
${ }^{a} h$, film thickness (nm).

${ }^{b} \lambda_{\text {DFB }}$, DFB emission wavelength (nm).

${ }^{c} \lambda_{\mathrm{ASE}}$, ASE emission wavelength (nm).

${ }^{d} I_{\text {th-DFB }}$, pump intensity threshold for DFB observation $(\mu \mathrm{J} /$ pulse $)$

${ }^{e} I_{\text {th-ASE }}$, pump intensity threshold for ASE observation ( $\mu \mathrm{J} /$ pulse).
} 


\section{Conclusions}

Second-order DFB lasers based on PS films doped with $30 \mathrm{wt} . \%$ of the blue-emitting and holetransporting organic molecule TPD as gain media have been fabricated and their laser properties under optical pumping have been evaluated. Gratings with a period of $270 \mathrm{~nm}$ and a depth of $70 \mathrm{~nm}$ were fabricated on glass by holographic lithography and subsequent etching methods. Laser thresholds of $6 \mu \mathrm{J} /$ pulse and linewidths lower than $2 \mathrm{~nm}$ were measured. The benefits of the presence of the grating in improving the laser performance were evidenced by comparing with films without gratings, which showed ASE thresholds 2 times larger and linewidths of around $5 \mathrm{~nm}$. The emission wavelength of the DFB structures was tuned in the range of $415-427 \mathrm{~nm}$ by changing the thickness of the active film. The fact that laser emission is observed in all cases on the long-wavelength edge of the stop band points to a system dominated by index modulation, rather than by gain modulation.

The authors acknowledge support from the Spanish Ministerio de Educación y Ciencia (MEC), the European Community (FEDER) (grants MAT200507369-C03-01 and MAT2008-06648-C02-01) and the University of Alicante (grant VIGROB2005060). We also thank V. Esteve for technical assistance.

\section{Reference}

1. F. Hide, B. J. Schwartz, M. A. Díaz-García, and A. J. Heeger, "Laser emission from solutions and films containing semiconducting polymers and titanium dioxide nanocrystals," Chem. Phys. Lett. 256, 424-430 (1996).

2. F. Hide, M. A. Diaz-Garcia, B. J. Schwartz, M. Andersson, Q. Pei, and A. J. Heeger, "Semiconducting polymers: a new class of solid-state laser materials," Science 273, 1833-1836 (1996).

3. N. Tessler, G. J. Denton, and R. H. Friend, "Lasing from conjugated-polymer microcavities," Nature 382, 695-697 (1996).

4. M. D. McGehee and A. J. Heeger, "Semiconducting (conjugated) polymers as materials for solid-state lasers," Adv. Mater. 12, 1655-1668 (2000) and references therein.

5. N. Tessler, "Lasers based on semiconducting organic materials," Adv. Mater. 11, 363-370 (1999) and references therein.

6. I. D. W. Samuel and G. A. Turnbull, "Organic semiconductor lasers," Chem. Rev. 107, 1272-1295 (2007) and references therein.

7. F. J. Duarte and L. W. Hillman, eds., Dye Laser Principles with Applications (Academic, 1990).

8. G. A. Turnbull, P. Andrew, W. L. Barnes, and I. D. W. Samuel, "Operating characteristic of a semiconducting polymer laser pumped by a microchip laser,” Appl. Phys. Lett. 82, 313-315 (2003).

9. A. E. Vasdekis, G. E. Town, G. A. Turnbull, and I. D. W. Samuel, "Fluidic fibre dye laser," Opt. Express 15, 3962-3967 (2007).

10. A. E. Vasdekis, S. A. Moore, A. Ruseckas, T. F. Krauss, I. D. W. Samuel, and G. A. Turnbull, "Silicon based organic semiconductor laser," Appl. Phys. Lett. 91, 051124 (2007).

11. M. D. McGehee, R. Gupta, S. Veenstra, E. K. Miller, M. A. Díaz-García, and A. J. Heeger, "Amplified spontaneous emission from photopumped films of a conjugated polymer," Phys. Rev. B 58, 7035-7039 (1998).

12. M. A. Díaz-García, S. Fernández De Avila, and M. G. Kuzyk. "Dye-doped polymers for blue organic diode lasers," Appl. Phys. Lett. 80, 4486-4488 (2002).
13. M. A. Díaz-García, E. M. Calzado, J. M. Villalvilla, P. G. Boj, J. A. Quintana, F. Giacalone, J. L. Segura, and N. Martín, "Concentration dependence of amplified spontaneous emission in two oligo-( $p$-phenylenevinylene)," J. Appl. Phys. 97, 063522 (2005).

14. E. M. Calzado, J. M. Villalvilla, P. G. Boj, J. A. Quintana, R. Gómez, J. L. Segura, and M. A. Díaz-García, "Amplified spontaneous emission in polymer films doped with a perylenediimide derivative," Appl. Opt. 46, 3836-3842 (2007).

15. E. M. Calzado, J. M. Villalvilla, P. G. Boj, J. A. Quintana, R. Gómez, J. L. Segura, and M. A. Díaz-García, "Effect of structural modifications in the spectral and laser properties of perylenediimide derivatives," J. Phys. Chem. C 111, 13595 (2007).

16. G. Gigli, G. Barbarella, L. Favaretto, F. Cacialli, and R. Cingolani, "High-efficiency oligothiopene-based light-emitting diodes," Appl. Phys. Lett. 75, 439-441 (1999).

17. N. Johansson, J. Salbeck, J. Bauer, F. Weissörtel, P. Bröms, A. Andersson, and W. R. Salaneck, "Solid-state amplified spontaneous emission in some spiro-type molecules: A new concept for the design of solid-state lasing molecules," Adv. Mater. 10, 1137 (1998).

18. S. Lattante, M. Anni, M. Salerno, L. Lagonigro, R. Cingolani, G. Gigli, M. Pasini, S. Destri, and W. Porzio, "Optical gain in fluorenyl-thiophene co-oligomer thin films," Opt. Mater. 28, 1072-1075 (2006).

19. S. Lattante, M. De Giorgi, G. Barbarella, and L. Favaretto, "Interplay between stimulated emission and singlet-singlet annihilation in oligothiophene dioxide thin films," J. Appl. Phys. 100, 023530 (2006).

20. J. Thomson, M. Anni, S. Lattante, D. Pisignano, R. I. R. Blyth, G. Gigli, and R. Cingolani, "Amplified spontaneous emission in the near infrared from a dye-doped polymer thin film," Synth. Met. 143, 305-307 (2004).

21. T. Maillou, J. Le Moigne, V. Dumarcher, L. Rocha, B. Geffroy, and J.-M. Nunzi, "Oligo (phenyl-ethynylene) as high photoluminescence quantum yield materials and its distributed feedback (DFB) laser emission properties in thin films," Adv. Mater. 14, 1297-1301 (2002).

22. X. Zhu, D. Gindre, N. Mercier, P. Frère, and J.-M. Nunzi, "Amplified stimulated emission from a needle-like single crystal of an end-capped fluorene/phenylene co-oligomer," Adv. Mater. 15, 906-909 (2003).

23. A. Costela, I. García-Moreno, and R. Sastre, "Polymeric solidstate dye lasers: recent developments," Phys. Chem. Chem. Phys. 5, 4745-4763 (2003).

24. W. Holzer, A. Penzkofer, and H.-H. Hörhold, "Travelling-wave lasing of TPD solutions and neat films," Synth. Met. 113, 281-287 (2000).

25. M. A. Díaz-García, E. M. Calzado, J. M. Villalvilla, P. G. Boj, J. A. Quintana, and M. G. Kuzyk, "TPD-based blue organic lasers," J. Nonlinear Opt. Phys. Mater. 13, 621 (2004).

26. E. M. Calzado, J. M. Villalvilla, P. G. Boj, J. A. Quintana, and M. A. Díaz-García, "Tuneability of amplified spontaneous emission through control of the thickness in organic-based waveguides,” J. Appl. Phys. 97, 093103 (2005).

27. E. M. Calzado, J. M. Villalvilla, P. G. Boj, J. A. Quintana, and M. A. Díaz-García, "Concentration dependence of amplified spontaneous emission in organic-based waveguides," Org. Electron. 7, 319 (2006).

28. K. Bando, T. Nakamura, and Y. Masumoto, "Origin of the amplified spontaneous emission from thiophene/phenylene co-oligomer single crystals: towards co-oligomers lasers," J. Appl. Phys. 99, 013518-013521 (2006).

29. W. Xie, Y. Li, F. Li, F. Shen, and Y. Ma, "Amplified spontaneous emission from cyano-substituted oligo ( $p$-phenylene vinylene) single crystal with very high photoluminescence efficiency," Appl. Phys. Lett. 90, 141110 (2007). 
30. J. C. Ribierre, G. Tsiminis, S. Richardson, G. A. Turnbull, I. D. W. Samuel, H. S. Barcena, and P. L. Burn, "Amplified spontaneous emission and lasing properties of bisfluorene-cored dendrimers," Appl. Phys. Lett. 91, 081108 (2007).

31. R. Xia, G. Heilotis, Y. Hou, and D. D. C. Bradley, "Fluorenebased conjugated polymer optical gain media," Org. Electron. 4, 165-177 (2003).

32. L. A. Coldren and S. W. Corzine, Diode Lasers and Photonic Integrated Circuits (Wiley 1995).

33. M. D. McGehee, M. A. Díaz-García, F. Hide, R. Gupta, E. K. Miller, D. Moses, and A. J. Heeger, "Semiconducting polymer distributed feedback laser," Appl. Phys. Lett. 72, 1536-1538 (1998).

34. G. A. Turnbull, P. Andrew, M. J. Jory, W. L. Barnes, and I. D. W. Samuel, "Relationship between photonic band structure and emission characteristic of a polymer distributed feedback laser," Phys. Rev. B 64, 125122 (2001).

35. J. A. Quintana, P. G. Boj, J. Crespo, J. A. Vallés-Abarca, and J. M. Villalvilla, "Diffraction gratings in dry developed dichromated gelatin films," Thin Solid Films 317, 343-346 (1998).

36. J. M. Villalvilla, J. Crespo, J. A. Quintana, C. Santos, and J. A. Vallés-Abarca, "Oxygen ECR stream etching of dichromated gelatin films," Thin Solid Films 317, 340-342 (1998).

37. V. Dumarcher, L. Rocha, C. Denis, C. Fiorini, J.-M. Nunzi, F. Sobel, B. Sahraoui, and D. Gindre, "Polymer thin-film distributed feedback tunable lasers," J. Opt. A Pure Appl. Opt. 2, 279-283 (2000).

38. G. Heliotis, R. Xia, G. A. Turnbull, P. Andrew, W. L. Barnes, I. D. W. Samuel, and D. D. C. Bradley, "Emission characteristics and performance comparison of polyfluorene lasers with one- and two-dimensional distributed feedback," Adv. Funct. Mater. 14, 91-97 (2004).

39. H. Kogelnik and C. V. Shank, "Couple-wave theory of distributed feedback lasers,” J. Appl. Phys. 43, 2327-2335 (1972).
40. G. A. Turnbull, P. Andrew, W. L. Barnes, and I. D. W. Samuel, "Photonic mode dispersion of a two-dimensional distributed feedback polymer laser," Phys. Rev. B 67, 165107 (2003).

41. G. Heliotis, R. Xia, and D. D. C. Bradley, "Blue, surfaceemitting distributed feedback polyfluorene lasers," Appl. Phys. Lett. 83, 2118-2121 (2003).

42. S. Riechel, U. Lemmer, J. Feldmann, T. Benstem, W. Kowalsky, U. Scherf, A. Gombert, and V. Wittwer, "Laser modes in organic solid-state distributed feedback lasers," Appl. Phys. B. 71, 897-900 (2000).

43. C. Bauer, H. Giessen, B. Schnabel, E. B. Kley, C. Schmitt, U. Scherf, and R. F. Mahrt, "A surface-emitting circular grating polymer laser," Adv. Mater. 13, 1161-1164 (2001).

44. I. Silvestre, P. W. B. Marques, M. Valadares, and L. A. Cury, "Gain coefficient method for amplified spontaneous emission in thin waveguided film of a conjugated polymer," Appl. Phys. Lett. 93, 163307 (2008).

45. N. Tsutsumi, T. Kawahira, and W. Sakai, "Semiconducting polyfluorenes as materials for solid-state polymer lasers across the visible spectrum," Appl. Phys. Lett. 83, 2533-2535 (2003).

46. R. Xia, G. Heliotis, and D. D. C. Bradley, "Amplified spontaneous emission and distributed feedback lasing from a conjugated compound in various polymer matrices," Synth. Met. 140, 117-120 (2004).

47. R. Xia, G. Heliotis, and D. D. C. Bradley, "Fluorene-based polymer gain media for solid-state laser emission across the full visible spectrum,” Appl. Phys. Lett. 82, 3599-3601 (2003).

48. V. Trabadelo, A. Juarros, A. Retolaza, M. G. Ramírez, V. Navarro-Fuster, J. M. Villalvilla, P. G. Boj, J. A. Quintana, and M. A. Díaz-García, "Highly photostable solid-state organic distributed feedback laser fabricated via thermal nanoimprint lithography," Microelectron. Eng., doi:10.1016/j.mee.2009. 11.142 (in press). 